\title{
Closed-Loop Planar Motion Control of a Steerable Probe with a "Programmable Bevel" Inspired by Nature
}

\author{
Seong Young Ko, Member, IEEE, Luca Frasson, and Ferdinando Rodriguez y Baena, Member, IEEE
}

\begin{abstract}
Percutaneous intervention has attracted significant interest in recent years, but many of today's needles and catheters can only provide limited control of the trajectory between an entry site and soft tissue target. In order to address this fundamental shortcoming in minimally invasive surgery, we describe the first prototype of a bio-inspired multi-part probe able to steer along planar trajectories within a compliant medium by means of a novel "programmable bevel", where the steering angle becomes a function of the offset between interlocked probe segments. A kinematic model of the flexible probe and programmable bevel arrangement is derived. Several parameters of the kinematic model are then calibrated experimentally with a fully functional scaled-up prototype, which is $\mathbf{1 2} \mathbf{~ m m}$ in diameter. A closed-loop control strategy with feed-forward and feedback components is then derived and implemented in vitro using an approximate linearization strategy first developed for car-like robots. Experimental results demonstrate satisfactory twodimensional trajectory following of the prototype $(0.68 \mathrm{~mm}$ tracking error, with $1.45 \mathrm{~mm}$ STD) using an electromagnetic position sensor embedded at the tip of the probe.
\end{abstract}

Index Terms-Biologically inspired robots, closed-loop control, medical robots and systems, needle steering, nonholonomic motion planning

\section{INTRODUCTION}

$\mathrm{P}$ ERCUTANEOUS intervention has always attracted significant interest because it is performed through the skin and, as such, it has several advantages for the patient [1]. Tumor biopsy, brachytherapy, deep brain stimulation and localized drug delivery, for instance, benefit from this operative technique to reduce tissue trauma and hospitalization time.

In order to localize a lesion, preoperative planning using Computer Tomography (CT) or Magnetic Resonance (MR)

Manuscript received May 14, 2010. This work was supported by the EUFP7 Project ROBOCAST (FP7-ICT-215190) and has also received funding from the European Research Council under the European Union's Seventh Framework Programme (FP7/2007-2013) / ERC grant agreement ${ }^{\circ}[258642-$ STING].

S. Y. Ko and L. Frasson are with Department of Mechanical Engineering, Imperial College London, SW7 2AZ, UK (e-mail: s.ko@imperial.ac.uk, luca.frasson07@imperial.ac.uk).

F. Rodriguez y Baena is with Department of Mechanical Engineering and the Institute of Biomedical Engineering, Imperial College London, SW7 2AZ, UK (corresponding author to provide phone: +44-(0)20-7594-7046; fax: +44(0)20-7594-1472; e-mail: f.rodriguez@imperial.ac.uk). images is often necessary. If the lesion is reachable through a straight path, a straight rigid needle can be used. For a safe operation, the location of the needle tip can be monitored by means of external markers mounted on the proximal end of the needle by relying on the fixed geometrical relationship between the base and needle point.

Recently, there have been efforts to introduce steerable needles in percutaneous interventions where a straight path does not seem possible or is not safe. Steerable needles are generally very flexible and can thus bend during tissue penetration. In principle, this feature could enable a surgeon to take a "roundabout" way to a target if "no-go" areas, for instance important vessels, are encountered along the straight line path intersecting entry and target locations. A suitable steering strategy, however, needs to be developed to exploit the flexibility of the needle, and localization of the tip position becomes more difficult since it is no longer possible to extrapolate tip position from a base measurement.

Three main approaches to the needle steering problem have been proposed to date. By modeling the material and geometric properties of needles and their behavior in soft tissue, DiMaio and Salcudean developed a model-based trajectory planner, where needle deflection and the soft tissue's deformation are used as a means to predict tip orientation in a compliant medium [2]. For real-time simulation and path planning, Glozman and Shoham subsequently developed a needle steering algorithm using a simpler model for the needle and the soft tissue, where the model considered springs to predict needle-tissue interaction forces [3]. These approaches take into account the deflection of a relatively stiff needle, which can be controlled by applying a suitable combination of moments at its base.

The second approach to needle steering relies upon the concept of preloaded concentric tubes, which are able to slide with respect to each other to produce curvilinear configurations $[4,5]$. By modeling the kinematics and dynamics properties of these nested segments, accurate tip motion control in three-dimensions has been demonstrated [6], while path tracking along curvilinear trajectories within a compliant medium (i.e. not within a lumen or cavity) has not yet been achieved.

The third and final approach to the needle steering problem exploits a thin and flexible needle with a bevel tip. In this embodiment, the asymmetry of the needle tip is exploited to 
produce a curved path within tissue, which can be controlled by altering the bevel angle and area, the needle material and its cross-sectional diameter. On this basis, Webster III et al. proposed a kinematic model of a bendable needle with a fixed bevel tip in three-dimensional space [7]. Alterovitz et al. derived a motion-planning algorithm in the presence of measurement uncertainty to obtain better targeting accuracy for a bevel-tip flexible needle [8]. Reed et al. modeled the torsional dynamics of a flexible needle to analyze torsional behavior during needle insertion [9]. These studies focus on open-loop trajectory control based on a kinematic model of the probe, coupled with a probe-tissue interaction model.

Indeed, the control of flexible needles represents a current research focus, with several attempts being published in the literature which have substantially advanced the state of the art. Kallem and Cowan proposed a plane alignment control algorithm for needle steering along planar trajectories to minimize the off-plane error [10]. They utilized a stereo camera to measure the tip position of a flexible needle and implemented a full-state observer to estimate missing states (such as the rotational degrees of freedom of the needle, which could not be explicitly measured). In order to generate multiple trajectories with a single flexible probe with a fixed bevel tip, Minhas et al. [11, 12] and Wood et al. [13] proposed a duty-cycling spinning algorithm - by periodically changing the orientation of the needle along its long axis by means of a base-mounted revolute actuator, trajectories with different radii could be achieved. In order to reduce drilling effects during the duty-cycle spinning of a beveled needle, Hauser et $a l$. proposed an algorithm using variable helical paths, based on the principle that the flexible needle will generate a helical path during simultaneous rotation and insertion [14].

In the presence of significant uncertainty, for instance that introduced by complex deformations of a soft tissue under dynamic load, closed-loop control is required to keep the needle on a predefined trajectory while subjected to dynamic loading conditions. In closed-loop feedback control, an external sensing device, whether incorporated into the needle tip (e.g. the electromagnetic sensor described further in this paper) or available during the insertion process (e.g. intraoperative fluoroscopy), is required to monitor the actual tip position within the substrate. To our knowledge, only two demonstrations of the latter approach (i.e. with image-guided feedback control) are reported in the literature, while the use of an embedded sensor to steer a needle has remained an open research challenge until now. Specifically, Glozman and Shoham utilized fluoroscopic images to measure the deflection of a stiff needle (without bevel tip) when inserted into tissue [15]. In Reed et al. [16], a position estimator based on stereo camera images is used to steer a thin and flexible bevel tip needle into gelatin. An "on-off" controller, which switches between "bevel-right" and "bevel-left", is complemented by a path planning module, torsion compensation and an off-plane error minimization algorithm. These approaches have been generally successful, but rely upon external sensors and complex image processing which limit the range of viable applications for this type of technology.
Recent works also demonstrated possible clinical applications of these methods. Majewicz et al. demonstrated three potential clinical applications of needle steering in $e x$ vivo tissue simulations: ablation, biopsy and brachytherapy [17]. Burdette et al. have integrated a two-segment concentric tube with an ultrasonic-based ablator, performing multiple thermal ablation in ex vivo bovine liver under threedimensional ultrasound monitoring. With a single penetration, by retracting and pushing only one of the nested tubes, three different tissue locations were ablated [18].

This paper describes the design, implementation and control of a flexible multi-part probe inspired by the ovipositor, or egg-laying channel, of certain insects, the foundations for which have previously been published [19-22]. The probe, which is composed of four interlocked probe segments, is able to alter its direction by means of a "programmable bevel tip", which is described here, alongside the development of a control strategy to drive the probe along planar trajectories within a compliant medium (gelatin). A small electromagnetic (EM) position sensor, embedded within the tip of the probe, is employed to monitor the tip position and orientation, providing the command signal for a bespoke feedback controller developed for the probe. A significant advantage of this system is that it is expected to be able to follow arbitrary curvilinear trajectories without discontinuities. With the ability to smoothly change the orientation of the probe's tip without the need for torsion along the long axis of the probe, thanks to the programmable bevel concept, the probe is also expected to cause less strain on the surrounding tissue, with a consequent reduction in tissue damage. Possible future target applications include keyhole neurosurgery (e.g. implantation of deep brain stimulation electrodes) and tissue biopsy of inaccessible, deep seated regions of the body.

This paper is organized as follows: the biological inspiration for this work and the "programmable bevel" concept are outlined in Section II. Section III describes the kinematic model developed for the probe and bevel assembly. A bespoke control strategy for two-dimensional needle steering, built around the kinematic model in Section III, is explained in Section IV. Section V describes the experimental results obtained with a first $12 \mathrm{~mm}$ outer diameter (OD) proofof-concept prototype and also explains the calibration of important parameters related to the kinematic model. Finally, conclusions and future work are outlined in Section VI. As a point of note, this paper expands on a previous report [23], by covering the foundations of the biologically-inspired probe design, the implementation of a scaled-up $12 \mathrm{~mm}$ prototype, and the experimental evaluation of the control strategy outlined in [23] within an artificial brain-like medium (gelatin).

\section{BIOLOGICAL INSPIRATION}

\section{A. A Flexible Probe Inspired by Ovipositing Wasps}

Ovipositing wasps (Fig. 1a) and the unique approach they employ to penetrate different kinds of substrate in order to lay eggs are the source of inspiration for the novel flexible and 


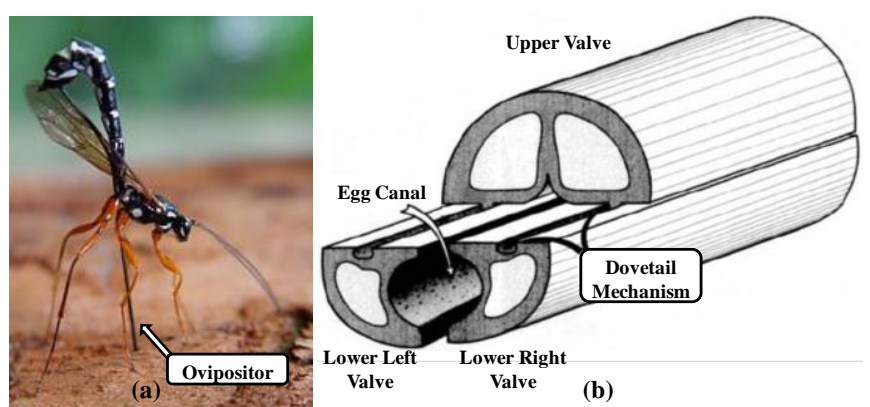

Fig. 1. (a) Giant ichneumon wasp Rhyssa persuasoria. Adult boring the surface of trunk infested with wood wasp larvae. Image courtesy of Boris Hrasovec, Faculty of Forestry. (b) Diagrammatic representation of oblique view of transversely cut ovipositor (modified from Quicke et al. 1995 [26])

steerable probe described here.

The ovipositor, a very long, thin and flexible structure [24, 25], consists of two or more segments (valves), which are connected by means of a special dovetail mechanism, as shown in Fig. 1b and are able to slide with respect to each other. An inner channel is used to deliver eggs in the substrate: some species lay eggs into the bark of wood, while parasitic species lay them into the soft tissue of hosts, such as larvae of other insects, often in a number of separate but adjacent locations. Even though the ovipositor is avoided of intrinsic muscular, the multi-part structure allows the reorientation of the ovipositor tip, which in turn enables it to steer within the substrate [26, 27].

Inspired by this natural design, a flexible probe potentially capable of three-dimensional steering in soft tissue is currently under development at Imperial College in London. A proof-ofconcept flexible probe prototype, composed of four segments connected to each other by means of an interlocking mechanism [21], is used to demonstrate probe steering. The current scaled-up prototype is not clinically viable, as a significantly smaller outer diameter (i.e. 1-4 mm OD) would be required for clinical deployment. However, ongoing work on the miniaturization the probe, the optimization of the probe's interlocking mechanism [22] and the modeling of the probe-tissue interaction forces [28], is expected to facilitate its applicability in the near future.

A unique feature of the design centers upon the relationship between the offset between interlocked probe segments and the steering angle of the probe, or "programmable bevel" concept, which provides the foundations for the control strategy described in this work.

\section{B. Bio-Inspired Programmable Bevel}

In the programmable bevel concept described in the following sections, two interlocked probe segments would be sufficient to steer the tip in a plane. The subdivision of the probe into four interlocked segments, however, stems from the need to stabilize the insertion process and enable the future extension of this work to three-dimensional steering. In the wider context of this work, a unique reciprocating insertion method is being investigated, where each probe segment is inserted one at a time, while the remaining, stationary segments act as "rails", helping to transfer the forward push from the back of the probe, along the long axis of the probe, to the tip. It is believed that this approach to probe insertion will minimize the amount of tissue deformation at the probe-tissue interface, with a consequent reduction in tissue damage. This research hypothesis is currently under investigation and does not relate directly to the work presented here, but provides justification for the 4-part embodiment of the probe described next.

A diagram illustrating the key features of the 4-part probe concept is illustrated in Fig. 2. Through numerous laboratory experiments, the offset between the two leading segments (i.e. segment I and segment III) has been shown to be related to the steering angle of the probe tip. It is believed that this phenomenon is related to the amount of unsupported length associated to the leading segment (or "steering offset" in Fig. 2), since it is likely to affect the deflection magnitude experienced by the segment as a result of tissue reaction forces during insertion. While an analytical description of this phenomenon is currently under investigation, Section V describes an experimental calibration process where the relationship between offset and steering angle of a prototype is found to be approximately linear, a finding which forms the basis of the planar motion control strategy presented in this paper.

Since only planar trajectories are considered, the four-part probe in Fig. 2 is modeled in two-dimensions only, disregarding the passive segments which simply follow the lagging steering segments (i.e. Segments II and IV).

\section{Kinematic Modeling}

In the following modeling description it is assumed that trajectories are defined in a plane and that the probe, which is aligned with the plane during initial setup, is composed of two identical segments. Webster III et al. showed that the kinematic model of a bevel tip needle could be considered to be similar to that of a bicycle model with a fixed steering angle [7]. In the case of our probe, the steering direction can also be altered using the offset between the two segments and

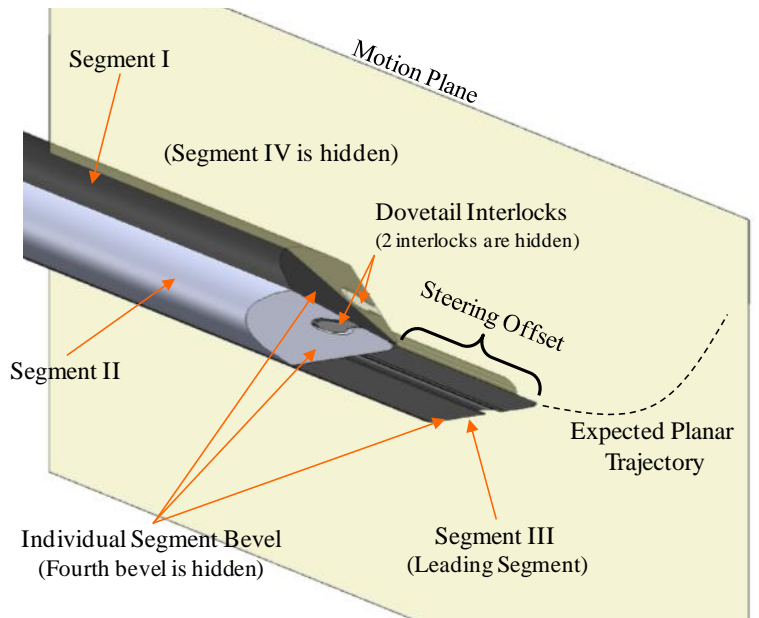

Fig. 2. Programmable bevel concept applied to a 4-part probe. Segments I and III define tip orientation (up or down), while segments II and IV are always aligned with the lagging steering segment (i.e. segment $\mathrm{I}$ in the diagram). 


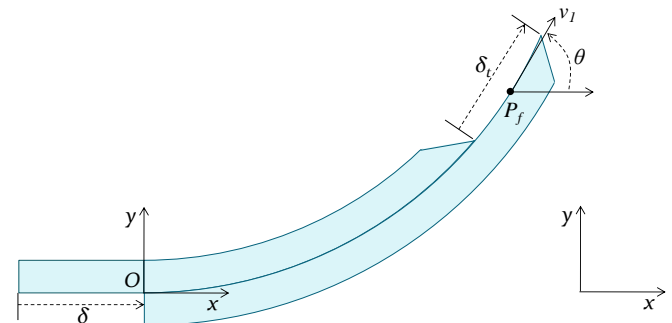

(a)

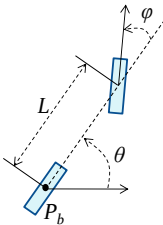

(b)
Fig. 3. Notation comparison between a flexible probe (a) and a conventional bicycle model (b). With the flexible probe, the curvature $\rho$ changes as a function of the steering offset $\delta_{t}$, while in the conventional bicycle model, $\rho$ is a function of the steering angle $\varphi$. In addition, the virtual tip position of the flexible probe $P_{f}$ is defined as the middle point between the two tips of the steering segments, while the corresponding point for the bicycle model $P_{b}$ is chosen to coincide with the rear wheel's center point. Also, the approach angle $\theta$ coincides with the orientation of the approach vector of the tip in the case of the flexible probe and the orientation of the body in the case of the bicycle model. $L$ indicates the length of the bicycle's body. Please note that the steering offset $\delta_{t}$ measured at the tip of the probe varies with respect to the offset $\delta$ measured at the base as function of the body configuration.

the kinematic model can thus be considered to be similar to that of a bicycle able to steer. Fig. 3a shows the notation adopted to describe the flexible probe, while Fig. 3b shows the notation associated with a conventional bicycle model [29]. If the origin of the bicycle model is chosen at the center of the rear wheel $\left(P_{b}\right)$, its kinematic model is expressed as follows:

$$
\left[\begin{array}{c}
\dot{x} \\
\dot{y} \\
\dot{\theta} \\
\dot{\varphi}
\end{array}\right]=\left[\begin{array}{c}
\cos \theta \\
\sin \theta \\
\tan \varphi / L \\
0
\end{array}\right] v_{1}+\left[\begin{array}{l}
0 \\
0 \\
0 \\
1
\end{array}\right] v_{2}
$$

where, $x, y, \theta$, and $\varphi$ indicate the $x$-axis and $y$-axis coordinates, the approach angle, and the steering angle of the bicycle model, respectively, and $v_{1}, v_{2}$, and $L$ indicate the forward velocity of a bicycle's body, the rate of change of the steering angle, and the distance between front and rear wheels, respectively.

In (1), the relationship between the forward velocity $\left(v_{l}\right)$ and the rotational velocity $(\dot{\theta})$ determines the instantaneous curvature $(\rho)$ of a real trajectory and it is a function of the steering angle $(\varphi)$, i.e. $\rho=\tan (\varphi) / L$ [10]. Contrary to the bicycle model, however, in our probe the instant curvature is assumed to be a function of the steering offset $\left(\delta_{t}\right)$ at the tip of the probe, as follows:

$\rho=f\left(\delta_{t}\right) \equiv \kappa \delta_{t}$

where, $f\left(\delta_{t}\right)$ is empirically assumed to be a monotonically increasing function of the steering offset.

In this research, we simplify the definition of $f\left(\delta_{t}\right)$ by treating the curvature $(\rho)$ as being proportional to the steering offset, with a coefficient $\kappa\left(\mathrm{mm}^{-2}\right)$, based on the calibration experiments described in Section V.C. Therefore, the kinematic model becomes as follows:

$$
\left[\begin{array}{c}
\dot{x} \\
\dot{y} \\
\dot{\theta} \\
\dot{\delta}_{t}
\end{array}\right]=\left[\begin{array}{c}
\cos \theta \\
\sin \theta \\
\kappa \delta_{t} \\
0
\end{array}\right] v_{1}+\left[\begin{array}{l}
0 \\
0 \\
0 \\
1
\end{array}\right] v_{t 2}
$$

In (3), $v_{l}$ and $v_{t 2}$ indicate the forward velocity and the changing rate of steering offset respectively and $x$ and $y$ indicate the $\mathrm{x}$ - and $\mathrm{y}$-axis coordinates of the virtual tip position of the flexible probe $P_{f}$. Note that the subscript " $t$ " in $v_{t 2}$ indicates that here the rate of change of steering angle is based on tip measurements (i.e. $\delta_{t}$ ).

The steering offset will in fact be different if measured at the base rather than the tip of the probe, due to a number of factors associated with this mechanism of motion e.g. axial compressive and tensile deformation of probe segments, probe body configuration and friction. Thus, let $\delta$ describe the offset between the two main segments of the probe, as illustrated in Fig. 3, while $\delta_{t}$ describes the corresponding offset, measured at the tip of the probe. Disregarding material deformation, a relationship between the two offsets which takes into account the probe's configuration can be derived as follows. Considering a flexible probe segment of infinitesimal length $d s$, as shown in Fig. 4, the curvature of the segment is $1 / R_{c}$ and the distance to a neutral axis for each segment is $r_{c}$. The length of each segment can be expressed as follows:

$\ell_{1}=\left(R_{c}-r_{c}\right) d \theta$

$\ell_{2}=\left(R_{c}+r_{c}\right) d \theta$

Given a segment with length $d s$, the angular difference between the two ends of the segment is $d \theta$, as in (5), and the difference between the two segments becomes $d \delta$, as in (6).

$d \theta=d s / R_{c}$

$d \delta=\ell_{2}-\ell_{1}=\left(R_{c}+r_{c}\right) d \theta-\left(R_{c}-r_{c}\right) d \theta$

$d \delta=2 r_{c} d \theta=\varepsilon d \theta$

By integrating both sides of (6), we can obtain a relationship between $\Delta \delta$ and the approach angle $\theta$ as follows:

$\Delta \delta=\varepsilon \Delta \theta=\varepsilon\left(\theta-\theta_{0}\right) \equiv \varepsilon \theta$

where $\varepsilon(\mathrm{mm})$ is the distance between the neutral axes of the two segments.

Assuming that the initial insertion direction for the probe is parallel to the $x$-axis (i.e. $\theta_{0}=0$ ), the compensation amount is thus only proportional to the current tip direction of the probe. To generate the correct steering offset $\delta_{t}$ at the tip of the probe, the prescribed offset imposed at the base, $\delta$, should thus be adjusted by $\Delta \delta$, as defined in (8).

$\delta=\delta_{t}+\Delta \delta$
$\delta_{t}=\delta-\Delta \delta=\delta-\varepsilon \theta$

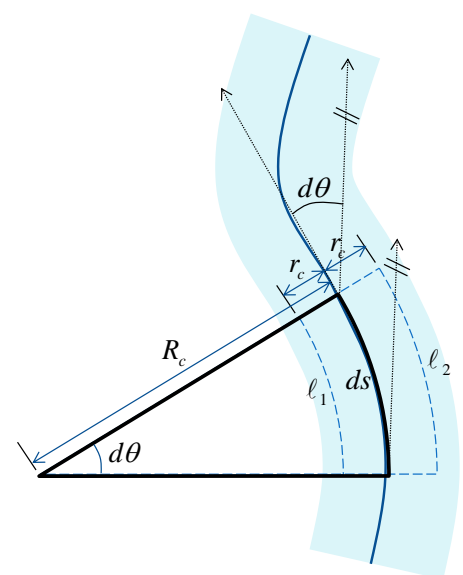

Fig. 4. Infinitesimal segment of the flexible probe having two segments. 


$$
\left[\begin{array}{c}
\dot{x} \\
\dot{y} \\
\dot{\theta} \\
\dot{\delta}
\end{array}\right]=\left[\begin{array}{c}
\cos \theta \\
\sin \theta \\
\kappa(\delta-\varepsilon \theta) \\
0
\end{array}\right] v_{1}+\left[\begin{array}{l}
0 \\
0 \\
0 \\
1
\end{array}\right] v_{2}
$$

A modified kinematic model of the flexible probe which takes into account this adjustment is described in (9), where $v_{l}$ and $v_{2}$ indicate the forward velocity and the changing rate of steering offset (with the offset applied at the base of the probe), respectively. The compensation coefficient $\varepsilon$ in (7) is determined theoretically as twice the distance from the center to the centroid of each segment [30]. $\varepsilon$ for a probe with two halves is thus $(8 R) /(3 \pi)$, and $(8 \sqrt{2} R) /(3 \pi)$ with four quarters. The kinematic model of the probe described in (9) is non-linear and can be expressed in the general form:

$\dot{q}=G(q) v$

where $q$ is a $(4 \times 1)$ vector of the flexible probe's generalized coordinates $[x, y, \theta, \delta]^{\mathrm{T}}, v$ is a $(2 \times 1)$ vector of input velocities $\left[v_{1}, v_{2}\right]^{\mathrm{T}}$, and the columns $g_{i}(i=1,2)$ of the $(4 \times 2)$ matrix $G(q)$ are vector fields [29].

\section{TWO-DIMENSIONAL TRAJECTORY FOLLOWING}

\section{A. Related Works and the Chained Form}

In order to construct a feedback control strategy for the probe, we adopt an algorithm developed for car-like robots $[29,31-36]$ which has received significant attention over the years and is generally expressed in the chained form representation [29, 31-32, 36]. In this method, the bicycle model, which is similar to the one developed here for the flexible probe, can be converted into the single chained form, which has two new control inputs $\left(u_{1}, u_{2}\right)$ and four new states $\left(\xi_{1}, \xi_{2}, \xi_{3}, \xi_{4}\right)$ as follows:

$\dot{\xi}_{1}=u_{1}$

$\dot{\xi}_{2}=u_{2}$

$\dot{\xi}_{3}=\xi_{2} u_{1}$

$\dot{\xi}_{4}=\xi_{3} u_{1}$

In order to obtain the relationships between the original coordinate $q$ and the new one $\xi$ and between the original input $v$ and the new one $u$, we can apply a similar approach to the one explained in [29]. First, by setting $\xi_{1}=x$ and comparing (9) and (11), the relations between $u_{l}$ and $v_{l}$ is obtained. Then, by setting $\xi_{4}=y, \xi_{3}$ and $\xi_{2}$ can be obtained using the original state and finally $u_{2}$ can be expressed using the original input. In this way, the kinematic model of the probe can be converted into the chained form, as illustrated in (12) and (13).

$$
\xi=\left[\begin{array}{l}
\xi_{1} \\
\xi_{2} \\
\xi_{3} \\
\xi_{4}
\end{array}\right]=\left[\begin{array}{c}
x \\
\kappa(\delta-\varepsilon \theta) / \cos ^{3}(\theta) \\
\tan (\theta) \\
y
\end{array}\right]=M(q)
$$

$$
\begin{aligned}
v & =\left[\begin{array}{cc}
\frac{1}{\cos \theta} & 0 \\
-\frac{3 \kappa(\delta-\varepsilon \theta)^{2} \sin \theta-\kappa \varepsilon(\delta-\varepsilon \theta) \cos \theta}{\cos ^{2} \theta} & \frac{\cos ^{3} \theta}{\kappa}
\end{array}\right]\left[\begin{array}{l}
u_{1} \\
u_{2}
\end{array}\right] \\
& =N(q) u
\end{aligned}
$$

In (12), the function $M$ converts the original coordinates $q$ into the chained form's coordinates $\xi$. Similarly, equation (13) describes the function $N$, which converts the chained form's input $u=\left[u_{1}, u_{2}\right]^{\mathrm{T}}$ back into the original input $v$.

Once the kinematics model of the flexible probe is converted into the chained form, feed-forward and feedback control become straightforward. First, based on a desired trajectory, feed-forward or feedback control input velocities can be computed using the chained form. Then, using (13), the inputs of the chained form can be converted back into the original inputs. The following sections (Sections IV.B and IV.C) reproduce many of the concepts described in [29], which have been included here for the sake of clarity.

\section{B. Feed-Forward Control}

Let us consider that a desired trajectory and its derivatives are given as in (14) and (15), respectively.

$$
\begin{aligned}
& x_{d}=x_{d}(\sigma(t)) \\
& y_{d}=y_{d}(\sigma(t)) \\
& \dot{x}_{d}(t)=\frac{d}{d t} x_{d}(t)=\frac{d x_{d}(\sigma)}{d \sigma} \frac{d \sigma}{d t}=x_{d}^{\prime}(\sigma) \dot{\sigma}(t) \\
& \dot{y}_{d}(t)=\frac{d}{d t} y_{d}(t)=\frac{d y_{d}(\sigma)}{d \sigma} \frac{d \sigma}{d t}=y_{d}^{\prime}(\sigma) \dot{\sigma}(t)
\end{aligned}
$$

Here, an intermediate trajectory parameter $\sigma$ is used to decouple the path description from the timing information as in [29] so as to define the trajectory independently of the desired forward velocity By defining $\xi_{d l}=x_{d}$ and $\xi_{d 4}=y_{d}$, the desired feed-forward control input can be obtained, as described in (16).

$u_{d 1}=x_{d}^{\prime}(\sigma) \dot{\sigma}(t)$

$u_{d 2}=\frac{x_{d}^{\prime 2} y_{d}^{\prime \prime \prime}-x_{d}^{\prime} x_{d}^{\prime \prime \prime} y_{d}^{\prime}-3 x_{d}^{\prime} x_{d}^{\prime \prime} y_{d}^{\prime \prime}+3 x_{d}^{\prime \prime 2} y_{d}^{\prime}}{x_{d}^{\prime 4}} \dot{\sigma}(t)$

In this case, the states of the probe will be as follows:

$\xi_{d}=\left[\begin{array}{l}\xi_{d 1} \\ \xi_{d 2} \\ \xi_{d 3} \\ \xi_{d 4}\end{array}\right]=\left[\begin{array}{c}x_{d} \\ \left(x_{d}^{\prime} y_{d}^{\prime \prime}-x_{d}^{\prime \prime} y_{d}^{\prime}\right) / x_{d}^{\prime 3} \\ y_{d}^{\prime} / x_{d}^{\prime} \\ y_{d}\end{array}\right]=L\left(x_{d}, y_{d}\right)$

In our application, the value $\dot{\sigma}(t)$ was utilized to keep the forward velocity $v_{d l}$ constant using (18).

$$
\begin{aligned}
& v_{d 1}(t)= \pm \sqrt{\dot{x}_{d}^{2}(t)+\dot{y}_{d}^{2}(t)}= \pm \sqrt{x_{d}^{\prime 2}(\sigma)+y_{d}^{\prime 2}(\sigma)} \dot{\sigma}(t) \\
& \dot{\sigma}(t)=v_{d 1}(t) / \sqrt{x_{d}^{\prime 2}(\sigma)+y_{d}^{\prime 2}(\sigma)}, \text { where } v_{d 1}(t) \geq 0
\end{aligned}
$$




\section{Feedback Control}

The feedback controller for trajectory tracking of the flexible probe is implemented here through approximate linearization [29]. This approach utilizes the state and input errors, which are denoted as in (19), to obtain the time-varying state space form.

$$
\begin{array}{ll}
\widetilde{\xi}_{i}=\xi_{d i}-\xi_{i}, & i=1, \ldots, 4 \\
\widetilde{u}_{j}=u_{d j}-u_{j}, & j=1,2
\end{array}
$$

The chained form in (11) can be represented using the nonlinear error equations as follows:

$$
\begin{aligned}
& \dot{\tilde{\xi}}_{1}=\tilde{u}_{1} \\
& \dot{\tilde{\xi}}_{2}=\tilde{u}_{2} \\
& \dot{\bar{\xi}}_{3}=\xi_{d 2} u_{d 1}-\xi_{2} u_{1}=u_{d 1} \widetilde{\xi}_{2}+\xi_{2} \tilde{u}_{1} \\
& \dot{\tilde{\xi}}_{4}=\xi_{d 3} u_{d 1}-\xi_{3} u_{1}=u_{d 1} \tilde{\xi}_{3}+\xi_{3} \widetilde{u}_{1}
\end{aligned}
$$

With the approximation $\xi_{2}=\xi_{d 2}$ and $\xi_{3}=\xi_{d 3}$, equation (20) can be converted into a linear state-space representation as in (21).

$$
\begin{aligned}
\dot{\xi} & =\left[\begin{array}{cccc}
0 & 0 & 0 & 0 \\
0 & 0 & 0 & 0 \\
0 & u_{d 1}(t) & 0 & 0 \\
0 & 0 & u_{d 1}(t) & 0
\end{array}\right] \widetilde{\xi}+\left[\begin{array}{cc}
1 & 0 \\
0 & 1 \\
\xi_{d 2}(t) & 0 \\
\xi_{d 3}(t) & 0
\end{array}\right] \tilde{u} \\
& =A(t) \tilde{\xi}+B(t) \tilde{u}
\end{aligned}
$$

where $\tilde{\xi}=\left[\begin{array}{llll}\tilde{\xi}_{1} & \tilde{\xi}_{2} & \tilde{\xi}_{3} & \tilde{\xi}_{4}\end{array}\right]^{T}$ and $\tilde{u}=\left[\begin{array}{ll}\tilde{u}_{1} & \tilde{u}_{2}\end{array}\right]^{T}$.

If input errors are defined as follows:

$$
\begin{aligned}
& \tilde{u}_{1}=-k_{1} \tilde{\xi}_{1} \\
& \tilde{u}_{2}=-k_{2} \tilde{\xi}_{2}-\frac{k_{3}}{u_{d 1}} \tilde{\xi}_{3}-\frac{k_{4}}{u_{d 1}} \tilde{\xi}_{4}
\end{aligned}
$$

The closed-loop system matrix of the probe becomes:

$$
A_{c l}(t)=\left[\begin{array}{cccc}
-k_{1} & 0 & 0 & 0 \\
0 & -k_{2} & -k_{3} / u_{d 1} & -k_{4} / u_{d 1}^{2} \\
-k_{1} \xi_{d 2} & u_{d 1} & 0 & 0 \\
-k_{1} \xi_{d 3} & 0 & u_{d 1} & 0
\end{array}\right]
$$

Equation (23) has the characteristic polynomial (24), the eigenvalues of which can be easily manipulated.

$\operatorname{det}\left(\lambda I-A_{c l}\right)=\left(\lambda+k_{1}\right)\left(\lambda^{3}+k_{2} \lambda^{2}+k_{3} \lambda+k_{4}\right)$

The overall control input to the chained form is thus:

$u=u_{d}-\tilde{u}$.

\section{Definition of Sensor-Specific Coordinates and Relations}

This section outlines the coordinate frames defined and used to measure the tip position of the flexible probe. Since the probe consists of four segments, but only one is tracked by means on an EM tracking sensor to minimize the size and complexity of the probe assembly, it is necessary to compute a relationship between the sensor data and the overall tip of the flexible probe. Fig. 5 depicts the coordinate frames adopted in this work in top-down view. Frame A, Frame B, Frame S, Frame $\mathrm{T}$ and Frame $\mathrm{F}$ denote the coordinate frames of the EM tracking system (in which EM sensor measurements are given), the base of the flexible probe, the EM sensor, the tip of the segment containing the sensor, and the virtual tip position of the flexible probe, respectively. All axes are defined parallel to each other at the outset.

Assuming the global frame of reference (or world coordinate system) to coincide with coordinate Frame B and provided that EM sensor position measurements are available in coordinate Frame A (i.e. ${ }^{A} T_{S}$ ), the tip position in base coordinates (i.e. ${ }^{B} T_{F}$ ) can be easily computed using (26).

$$
\begin{aligned}
{ }^{B} T_{F} & ={ }^{B} T_{A}{ }^{A} T_{S}{ }^{S} T_{T}{ }^{T} T_{F} \\
& =\left({ }^{A} T_{B}\right){ }^{-1} T_{S}{ }^{S} T_{T}{ }^{T} T_{F}
\end{aligned}
$$

where ${ }^{B} T_{F}$ represents the $4 \times 4$ transformation matrix of Frame $\mathrm{F}$ with respect to Frame B.

Here, ${ }^{B} T_{A}$ is assumed to become available by means of a suitable registration process (for instance via measurements obtained with a reference optical tracking system), ${ }^{S} T_{T}$ is defined as in (27), where ${ }^{s} x_{T}$ and ${ }^{s} y_{T}$ are obtained from measurements of the cross-sectional geometry of the probe, while ${ }_{z_{T}}$, which is affected by the chosen sensor position within the probe sensor channel (see Fig. 8), is obtained from physical measurements of the prototype, and ${ }^{T} T_{F}$ is computed using the process described in the following paragraphs.

$$
{ }^{s} T_{T}=\left[\begin{array}{cccc}
1 & 0 & 0 & { }^{s} x_{T} \\
0 & 1 & 0 & { }^{s} y_{T} \\
0 & 0 & 1 & { }^{s} z_{T} \\
0 & 0 & 0 & 1
\end{array}\right]=\left[\begin{array}{cccc}
1 & 0 & 0 & -3.9 \\
0 & 1 & 0 & 1.4 \\
0 & 0 & 1 & 10.9 \\
0 & 0 & 0 & 1
\end{array}\right]
$$

As described in Section II.B, two of the four segments, namely $S_{1}$ and $S_{3}$ in Fig. 5, are defined as steering segments. The remaining two segments, $S_{2}$ and $S_{4}$, are functionally passive, as they follow the lagging steering segment at all times. Thus, let us consider the situation in which there is an offset between the steering segments as shown in Fig. 6. Conforming to the notation outlined in Section III, the steering offset at the base $(\delta)$ and the corresponding offset at the tip $\left(\delta_{t}\right)$ can be computed as in (28) and (29), respectively, where $\theta$ defines the angle
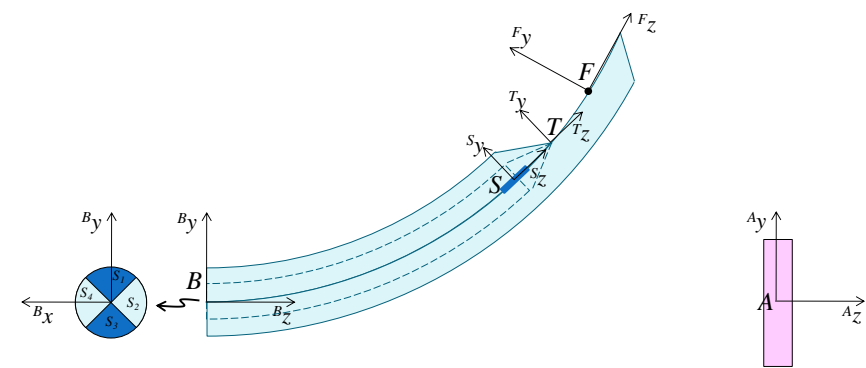

Fig. 5 Definition of coordinate systems for measuring the tip of a flexible probe. 
between ${ }_{Z}$ and ${ }^{B} Z$ and $\ell_{\mathrm{sx}}$ represents the length of segment "x".

$\delta=\ell_{S 3}-\ell_{S 1}$

$\delta_{t}=\delta-\delta \theta=\ell_{S 3}-\ell_{S 1}-\boldsymbol{\theta} \theta$

Based on Fig. 6, the angular difference between frame T and frame F (i.e. $\varphi_{f}$ ) can be computed as in (30) and (31), where $R_{c}$ is the signed radius of curvature for a given offset.

$$
\begin{aligned}
& \left|R_{c}\right| \varphi=\delta_{t} \\
& \left|R_{c}\right| \varphi_{f}=\delta_{t} / 2 \\
& \varphi_{f}=\frac{1}{\left|R_{c}\right|} \frac{\delta_{t}}{2}=\frac{|\rho| \delta_{t}}{2}=\frac{\left|\kappa \delta_{t}\right| \delta_{t}}{2}=\frac{\kappa \delta_{t}^{2} \operatorname{sgn}\left(\delta_{t}\right)}{2}
\end{aligned}
$$

${ }^{T} T_{F}$ can then be defined as follows:

$$
{ }^{T} T_{F}=\left[\begin{array}{cc}
{ }^{T} R_{F} & { }^{T} P_{F} \\
0^{T} & 1
\end{array}\right]
$$

where, ${ }^{T} P_{F}=\left[\begin{array}{lll}0 & R_{c}\left(1-\cos \varphi_{f}\right) & R_{c} \sin \varphi_{f}\end{array}\right]^{T}$ and

$$
{ }^{T} R_{F}=\left[\begin{array}{ccc}
1 & 0 & 0 \\
0 & \cos \varphi_{f} & \sin \varphi_{f} \\
0 & -\sin \varphi_{f} & \cos \varphi_{f}
\end{array}\right]
$$

The rotation matrix in (32) represents a rotation about $\varphi_{f}$ along the negative $x$-axis and $R_{c}$ has the same sign of $\delta_{t}$. Thus $R_{c} \sin \varphi_{f} \geq 0$.

In order to avoid the limiting condition of $R_{c}$ becoming infinite as the steering offset approaches zero, ${ }^{T} P_{F}$ is then approximated by the first three terms of an equivalent Taylor's series for the sine and cosine functions, as in (33):

$$
{ }^{T} P_{F}=\left[\begin{array}{c}
\kappa \delta_{t}^{3}\left(\frac{1}{2^{2} \cdot 2 !}-\frac{\kappa^{2} \delta_{t}^{4}}{2^{4} \cdot 4 !}+\frac{\kappa^{4} \delta_{t}^{8}}{2^{6} \cdot 6 !}\right) \\
\mid \delta_{t}\left(\frac{1}{2^{1} \cdot 1 !}-\frac{\kappa^{2} \delta_{t}^{4}}{2^{3} \cdot 3 !}+\frac{\kappa^{4} \delta_{t}^{8}}{2^{5} \cdot 5 !}\right)
\end{array}\right]
$$

\section{E. Control System Overview}

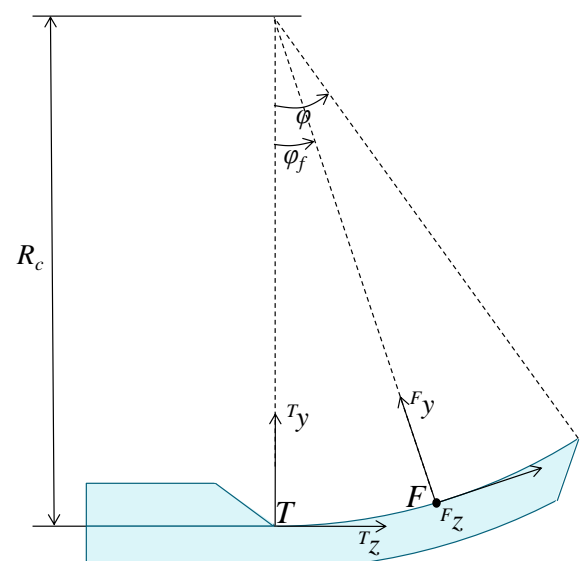

Fig. 6 Relationship between the virtual tip of a flexible probe and the tip of the segment containing the EM sensor.

Fig. 7 shows the overall block diagram of the closed-loop steering algorithm developed for the flexible probe. It has two main control loops: the local PID position controller for each probe segment and the steering controller, which implements trajectory following and consists of the feed-forward term (16), and the feedback term (22). In this research, the forward motion velocity $\left(v_{l}\right)$ and the rate of change of steering offset $\left(v_{2}\right)$ are arbitrarily defined as the average speed and the differential speed of the two steering segments $\left(S_{3}, S_{1}\right)$ respectively, as follows:

$v_{1}=\frac{\dot{\ell}_{S 3, d}+\dot{\ell}_{S 1, d}}{2}$
$v_{2}=\dot{\ell}_{S 3, d}-\dot{\ell}_{S 1, d}$

Segment lengths are calculated as in (35) and (36).

$\dot{\ell}_{S 1, d}=v_{1}-v_{2} / 2$

$\dot{\ell}_{S 3, d}=v_{1}+v_{2} / 2$

$\ell_{S 1, d}=\int \dot{\ell}_{S 1, d} d t$

$\ell_{S 3, d}=\int \dot{\ell}_{S 3, d} d t$

$\ell_{S 2, d}=\ell_{S 4, d}=\min \left(\ell_{S 1, d}, \ell_{S 3, d}\right)$

where $\ell_{d}=\left[\begin{array}{llll}\ell_{S 1, d} & \ell_{S 2, d} & \ell_{S 3, d} & \ell_{S 4, d}\end{array}\right]^{T}$.

A motion constraint is imposed on the computed output of

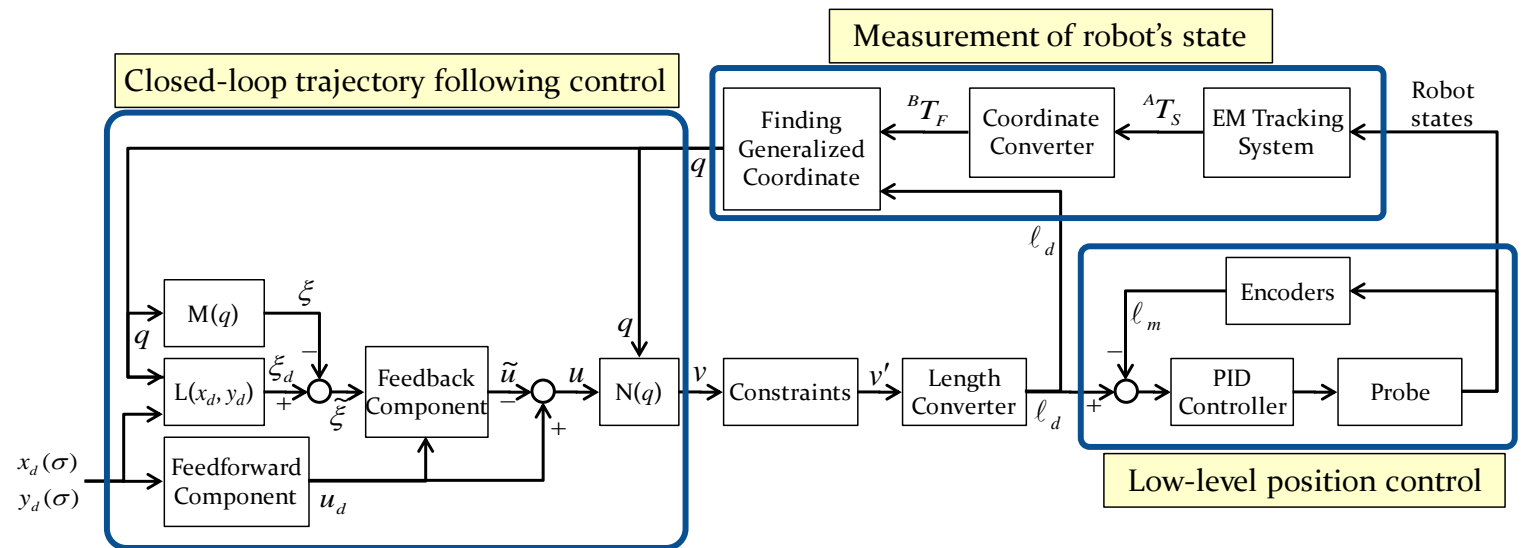

Fig. 7. Block diagram of the probe steering control strategy developed for the flexible probe. It includes a closed-loop trajectory controller, a low-level position controller for the robot actuators and measuring blocks for the robot's state 
the state controller to ensure that the command signals do not generate backward motion of any of the steering segments, as, in our probe embodiment, steering and insertion are inextricably linked (i.e. the probe tip cannot change orientation without further insertion). To ensure that the velocity of each segment remains equal to or greater than zero, the following constraint function (described with a "Constraints" block in Fig. 7) is included prior to the length conversion step:

$$
\begin{gathered}
\text { if }\left(v_{2}>2 v_{1}\right) \\
v_{2}=2 v_{1} \\
\text { else if }\left(v_{2}<-2 v_{1}\right) \\
v_{2}=-2 v_{1}
\end{gathered}
$$

Finally, the generalized coordinates $(q)$ of the flexible probe are obtained using ${ }^{B} T_{F}$ and $\ell_{d}$. For practical purposes, a simple transformation is included in the computation process, as described in (38), since the orientation of Frame O in Fig. 3 is different to that of Frame B in Fig. 5.

$$
\begin{aligned}
& { }^{o} T_{F}={ }^{o} T_{B}{ }^{B} T_{F} \\
& \text { where, }{ }^{o} T_{B}=\left[\begin{array}{cccc}
0 & 0 & 1 & 0 \\
0 & 1 & 0 & 0 \\
-1 & 0 & 0 & 0 \\
0 & 0 & 0 & 1
\end{array}\right]
\end{aligned}
$$

The generalized coordinate thus become:

$$
q=\left[\begin{array}{l}
x \\
y \\
\theta \\
\delta
\end{array}\right]=\left[\begin{array}{c}
o_{14} \\
o_{24} \\
\operatorname{atan} 2\left(o_{23}, o_{13}\right) \\
\ell_{S 3, d}-\ell_{S 1, d}
\end{array}\right]=\left[\begin{array}{c}
b_{34} \\
b_{24} \\
\operatorname{atan} 2\left(b_{23}, b_{33}\right) \\
\ell_{S 3, d}-\ell_{S 1, d}
\end{array}\right]
$$

where $o_{i j}$ and $b_{i j}$ are the $i^{\text {th }}$ row and $j^{\text {th }}$ column components of ${ }^{o} T_{F}$ and ${ }^{B} T_{F}$ respectively, and $\ell_{d}=\left[\ell_{S 1, d}, \ell_{S 2, d}, \ell_{S 3, d}, \ell_{S 4, d}\right]^{T}$.

\section{EXPERIMENTAL VALIDATION}

\section{A. Flexible Probe Prototype}

The key geometrical features of the flexible probe prototype developed for these experiments (length $=200 \mathrm{~mm}$, outer diameter $(\mathrm{OD})=12 \mathrm{~mm}$ ) are illustrated in Fig. 8, while Fig. 9 shows different view of the actual probe and actuation system assembly. The probe is composed of four segments connected together by means of a dovetail mechanism which allows sliding motion between the segments. Two of the segments are equipped with $1.9 \mathrm{~mm}$ diameter hollow channels, which run along the full length of the probe: one houses the EM sensor; the other acts as a general purpose working channel, which can for instance be used for suction or drug delivery.

The prototype is manufactured with rapid prototyping (RP) techniques in a rubber-like material with high elasticity (TangoBlack - FullCure 970, Objet; tensile strength of 2MPa; hardness of 61 Shore Scale A; elongation at break of $48 \%$ ).
The leftmost and rightmost extremities of each probe segment (Fig. 8a) are made out of a more rigid plastic material (VeroWhite - FullCure 830; tensile strength of $50 \mathrm{MPa}$; hardness of 83 Shore Scale D; elongation at break of 20\%) to improve the probe material toughness at stress points. The leftmost rigid part of each segment in Fig. 8a also features a $1.5 \mathrm{~mm}$ OD hole, which is used to secure the probe to a mechanical transmission cable, as shown in Fig. 9.

Each segment of the probe is controlled via a linear actuator assembly (DC motor plus lead screw arrangement: Maxon Amax 22 motor, $6 \mathrm{~W}$ power rating, $6.77 \mathrm{mNm}$ maximum continuous torque, 4.4 gear ratio, $4 \mathrm{~mm}$ pitch, $60 \%$ lead screw efficiency and $28.1 \mathrm{~N}$ calculated thrust force) connected to a transmission link with a diameter of $1.5 \mathrm{~mm}$, as shown in Fig. 9. The four linear actuators are packaged into a free-standing actuation box to improve handling and integration into the experimental setup. Fig. 9 shows the integrated probe prototype and actuation system.

\section{B. Experimental Setup}

Fig. 10 shows the experimental setup used to test the performance of the closed-loop trajectory controller described in Section IV with the $12 \mathrm{~mm}$ OD flexible probe prototype and a gelatin sample. The linear actuators are controlled via a compactRIO embedded controller programmed in Labview

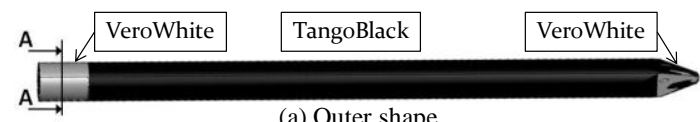

(a) Outer shape

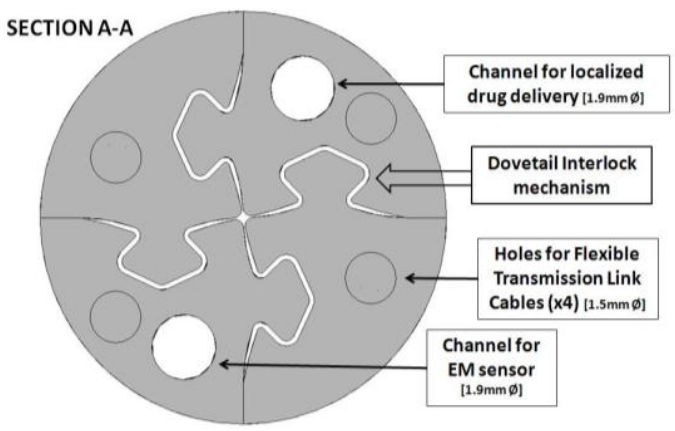

(b) Cross-sectional design

Fig. 8. Outer shape and cross-sectional design of flexible probe

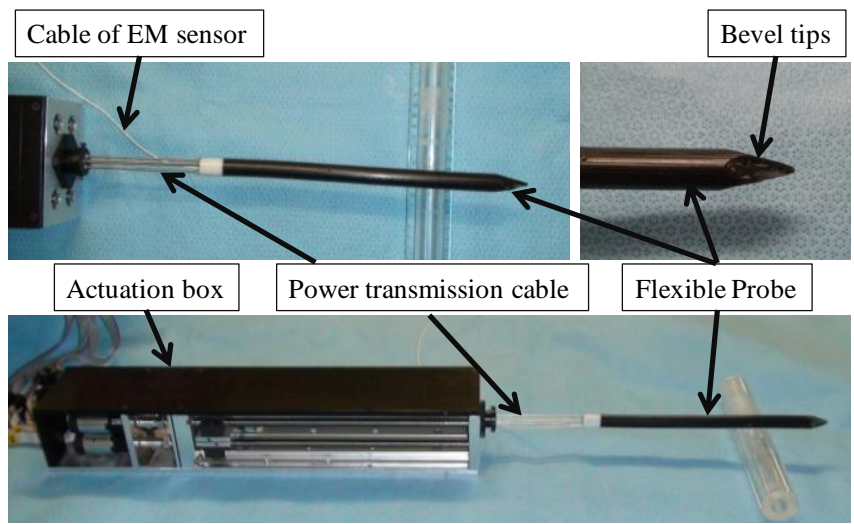

Fig. 9. Prototype of the $12 \mathrm{~mm}$ OD flexible probe and actuation box. 
(National Instruments inc.). A Labview-based graphical user interface (GUI) was developed and integrated into the setup in order to program desired trajectories, monitor performance and $\log$ key control parameters. An EM tracking sensor (Aurora 5DOF longlife sensor with $1.1 \mathrm{~mm}$ diameter, Northern Digital inc. Root-mean square (RMS) accuracy of $0.9 \mathrm{~mm} / 0.3^{\circ}$ [37]) was employed to measure the probe's tip position. The gelatin phantom (Fig. 10) was prepared with 6 weight $\%$, as used in previous work by Minhas et. al. [11] to mimic the insertion properties of biological soft tissue. A custom-made trocar, with $12.5 \mathrm{~mm}$ inner diameter, was also added to the setup to eliminate the possibility of buckling outside of the gelatin phantom. As a complement to the EM tracking data, all trajectories were also captured using a video camera (Sony Handycam HDR-SR10E) mounted on a static tripod in a topdown view arrangement.

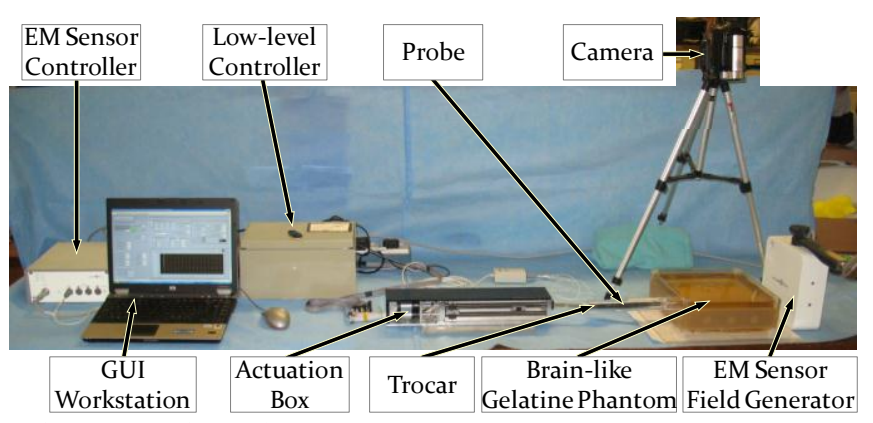

Fig. 10. Experimental setup
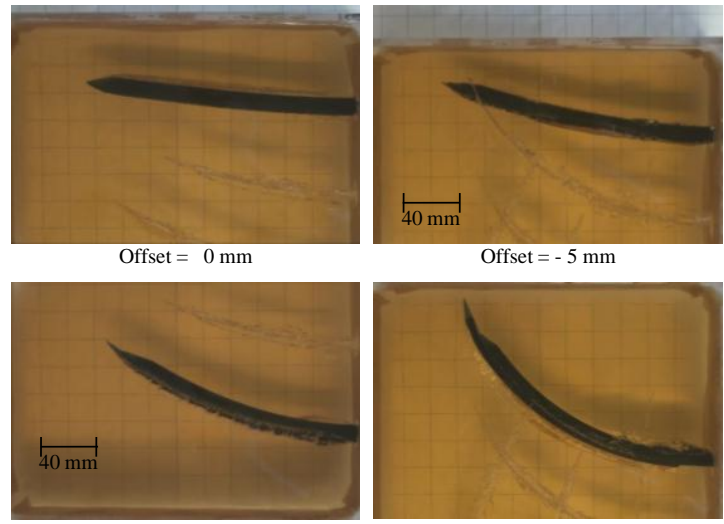

Offset $=-15 \mathrm{~mm}$

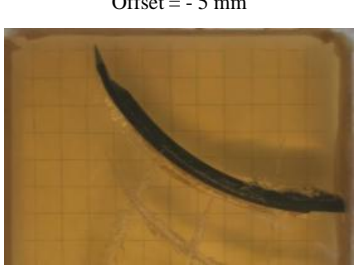

Offset $=-25 \mathrm{~mm}$

Fig. 11. Captured flexible probe trajectories for different steering offset values.

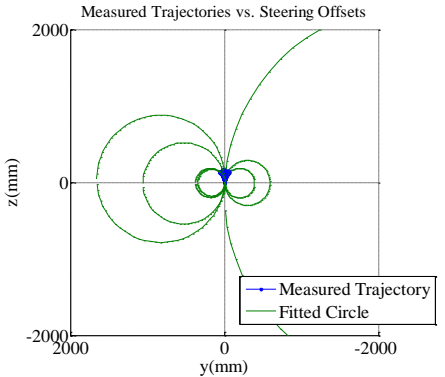

(a)

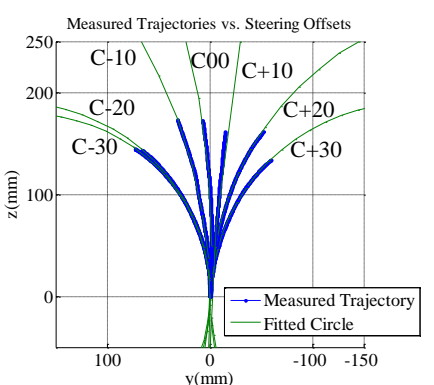

(b)
Fig. 12. Fitted trajectories: (a) best fit circles for each tested steering offset and (b) enlarged view of the trajectories achieved for offsets between $-30 \mathrm{~mm}$ and $30 \mathrm{~mm}$

\section{Steering Offset vs. Curvature Calibration}

Equation (2) describes the probe curvature as a function of the steering offset. In order to investigate this relationship, a set of experiments was performed. Ten simple insertion tests with constant steering offset $(\delta)$ of $-30,-25,-20,-15,-10,-5$, $0,10,20$ and $30 \mathrm{~mm}$ were performed (note that offset sign relates to steering direction). The trajectories of the tip of the segment containing the EM sensor $\left({ }^{B} P_{T}\right)$ were gathered using EM tracking measurements, while the overall shape of the curved probe was captured using the video camera system and processed through a manual segmentation process implemented in MATLAB. Each test started at rest, with all four segments aligned and embedded $1 \mathrm{~cm}$ into the gelatin sample. The leading steering segment $\left(S_{3}\right.$ for a positive offset and $S_{1}$ for a negative offset) was then driven further into the tissue by the required amount. Finally, all four segments were pushed together into the sample at a constant speed of $1 \mathrm{~mm} / \mathrm{second}$ down to a depth of approximately $15 \mathrm{~cm}$. Fig. 11 shows a few sample pictures captured by the camera. After each test, trajectory data measured by the EM tracking system for each test was first projected onto plane of best fit (by simple least squares fitting), then fitted to a circle to find the average radius of curvature for each trajectory. Most of the trajectories and corresponding circles are plotted in Fig. 12a, while Fig. 12b shows an enlarged view of the same. On the basis of these measurements, curvature values (i.e. the inverse of the radii of curvature) for each offset were computed and are plotted in Fig. 13. As can be seen, the curvature was found to be approximately proportional to the steering offset, with a coefficient $\kappa=1.85 \times 10^{-4} \mathrm{~mm}^{-2}\left(R^{2}=0.976\right)$ and the

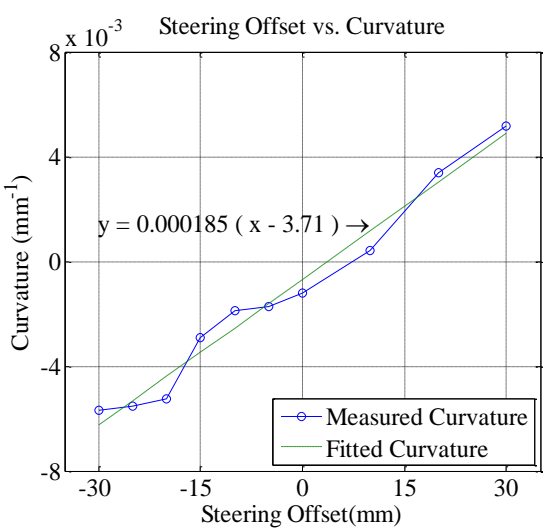

Fig. 13. Relationship between offset and curvature with linear fit.

TABLE 1

PARAMETERS USED FOR TRAJECTORY FOLLOWING EXPERIMENT

\begin{tabular}{|c|c|c|}
\hline & Values \\
\hline $\begin{array}{c}\text { Parameters } \\
\text { Ideal initial nosture }\end{array}$ & {$\left[x_{0}, y_{0}, \theta_{0}, \delta_{0}\right]^{\mathrm{T}}$} & {$\left[0 \mathrm{~mm}, 0 \mathrm{~mm}, 0^{\circ}, \delta_{\text {o, ideal }} \mathrm{mm}\right]^{\mathrm{T}}$} \\
\hline Initial posture disturbance & {$\left[x_{0}, y_{0}, \theta_{0}, \delta_{0}\right]^{\mathrm{T}}$} & {$\left[0 \mathrm{~mm}, 0 \mathrm{~mm}, 0^{\circ}, 0 \mathrm{~mm}\right]^{\mathrm{T}}$} \\
\hline Linear velocity & $v_{d l}$ & $1 \mathrm{~mm} / \mathrm{s}$ \\
\hline Steering coefficient & $\kappa$ & $0.000185 \mathrm{~mm}^{-2}$ \\
\hline Compensation coefficient & $\varepsilon$ & $(8 \times \sqrt{ } 2 \times 6) /(3 \pi)=7.20 \mathrm{~mm}^{*}$ \\
\hline $\begin{array}{l}\text { Time constant of low pass } \\
\text { filter for EM sensor data }\end{array}$ & $\tau_{q}$ & $0.1 \mathrm{~s}$ \\
\hline Control gain & {$\left[k_{1}, k_{2}, k_{3}, k_{4}\right]$} & {$[0.1,0.2,0.02,0.001]$} \\
\hline
\end{tabular}

A $6 \mathrm{~mm}$ radius reflects the size of the flexible probe prototype used for validation (Section $\mathrm{V}$ ). 
maximum curvature using a $30 \mathrm{~mm}$ steering offset measured $0.0056 \mathrm{~mm}^{-1}$, which corresponds to a radius of curvature of $178.6 \mathrm{~mm}$. The slight asymmetry in curvature values between positive and negative offsets could stem from manufacturing inaccuracies, trocar alignment errors, probe deformation, etc. This, however, is accounted for by the small but finite $\mathrm{x}$-axis intercept of $3.71 \mathrm{~mm}$, as illustrated in Fig. 13. While the linear approximation adopted here represents a substantial simplification and will require further work, it was found to be sufficiently accurate to produce satisfactory trajectory following, as described in Section V.D. As a point of note, these experiments were performed by penetrating a homogeneous gelatin phantom with constant speed. Although the closed-loop controller described in Section IV is expected to mitigate the effect of modeling uncertainties (e.g. rate dependency and heterogeneity of the substrate), a detailed study of the contribution which the material properties of the surrounding tissue have on tracking performance will be the focus of future work.

\section{Trajectory Following Results}

This section reports on the experimental results obtained with the flexible probe prototype and 2D trajectory following controller described in Fig. 7. The parameters used in these experiments are summarized in TABLE 1 . The linear forward velocity was arbitrarily chosen to be $1 \mathrm{~mm} / \mathrm{sec}$ on the basis an estimate of the speed of manual insertion of a standard deep brain stimulation electrode provided by an expert neurosurgeon. Published literature [38] also recommends that the advancing or withdrawing of microelectrodes or other instruments should be no greater than $0.5 \mathrm{~mm} / \mathrm{sec}$ to reduce the risk of hemorrhage, which is grossly in line with the speed chosen for these experiments. In the tests, the initial generalized coordinates $q$ are set to $[0,0,0,0]^{\mathrm{T}}$. In TABLE 1 , $\delta_{0, \text { ideal }}$ indicates the steering offset for the probe. The control gains were set to $\left[k_{1}, k_{2}, k_{3}, k_{4}\right]=[0.1,0.2,0.02,0.001]$, with corresponding eigenvalues of $[-0.1,-0.1,-0.05(1 \pm i \sqrt{3})]$, on the basis of the simulated results presented in [23].

Eight tests were performed - six with the double bend trajectory (40) and two with the circular trajectory (41).

$$
\begin{aligned}
& x_{d}(\sigma)=\sigma \\
& y_{d}(\sigma)=\frac{A}{2}\left(1-\cos \left(\frac{\pi}{L} \sigma\right)\right) \\
& x_{d}(\sigma)=R \sin (k \sigma) \\
& y_{d}(\sigma)=R(1-\cos (k \sigma))
\end{aligned}
$$

The parameters pertaining to the trajectory of each experiment, alongside the experimental results obtained, are listed in TABLE 2. The last four columns of TABLE 2 report on the overall steering results, expressed as the mean, standard deviation, root mean square (RMS) and maximum positional error between the probe tip and predefined trajectory.

Fig. 14 graphically illustrates the results obtained three out of the six experiments: Ex04 and Ex06 show the results obtained for the double bend trajectory and Ex07 shows one example of single bend trajectory. Three figures in the first row display the tip position of the flexible probe $\left({ }^{\mathrm{B}} \mathrm{P}_{\mathrm{F}}\right)$ estimated using (26) and (33), the tip position of the segment containing the EM sensor $\left({ }^{B} \mathrm{P}_{\mathrm{T}}\right)$, and the desired trajectory. Three figures in the second row show the positional error of the estimated tip $\left({ }^{\mathrm{B}} \mathrm{P}_{\mathrm{F}}\right)$ from the desired curvilinear trajectory. The final shapes of the flexible probe, which were captured using a video camera arranged in top-down view, are shown in the last row. The green solid lines indicate the central axes of the final shapes of the probe, which were obtained by averaging the upper and lower edges at the interface between the probe and the gelatin sample. The yellow dotted line indicates the trace of the virtual probe tip, which was obtained by taking the midpoint between the two tips of the steering segments at $5 \mathrm{sec}$ intervals from start to end. The RMS distance errors between these two lines are $0.91 \mathrm{~mm}, 1.79 \mathrm{~mm}$ and $1.80 \mathrm{~mm}$ for Ex04, Ex06 and Ex07, respectively.

One of the major factors affecting the tracking errors reported in TABLE 2 seems to stem from the interaction between the tissue and the probe and between probe segments. In order to change the steering direction and, for instance, move left, the right segment needs to move further. In doing so, the friction between the two steering segments pushes the whole probe to left against the tissue. This undesired effect shifts the direction of the probe tip away from the desired trajectory, thus introducing tracking errors. Specifically, in Ex04 the probe tip trajectory is first shifted in the positive ydirection and subsequently in the negative y-direction, after a change in desired direction.

Another factor which has been identified to be responsible for these tracking inaccuracies relates to excessively small gains associated to the positional error $\left(k_{4}\right)$. In simulation [23], the probe is not affected by the interaction between segments, thus small control gains for the positioning error are sufficient. Conversely, in these experiments it was necessary to increase the control gains to overcome sources of uncertainty which were not considered during the kinematic modeling of the probe. From (12), it is apparent that the four states of the chained form roughly correspond to the $x$-axis position, the curvature of the trajectory, the direction of the trajectory and

\begin{tabular}{|c|c|c|c|c|c|c|c|c|}
\hline & & $L(\mathrm{~mm})$ & $A(\mathrm{~mm})$ & $\begin{array}{l}\delta_{0, \text { ideal }} \\
(\mathrm{mm})\end{array}$ & mean & $\begin{array}{c}p_{\text {error }}(\mathrm{mI} \\
\text { standard } \\
\text { deviation }\end{array}$ & $\begin{array}{l}\text { m) } \\
\text { RMS }\end{array}$ & $\max$ \\
\hline \multirow{7}{*}{$\begin{array}{c}\text { Double } \\
\text { bend }\end{array}$} & Ex01 & 150 & 15 & 17.783 & 0.10 & 0.45 & 0.46 & 1.29 \\
\hline & Ex02 & 150 & 15 & 17.783 & 0.24 & 0.98 & 1.01 & 1.75 \\
\hline & Ex03 & 150 & 20 & 23.711 & 0.52 & 0.75 & 0.91 & 1.74 \\
\hline & Ex04 & 150 & 20 & 23.711 & 0.55 & 1.45 & 1.55 & 2.72 \\
\hline & Ex05 & 150 & 25 & 29.638 & 0.15 & 1.43 & 1.44 & 2.35 \\
\hline & Ex06 & 150 & 25 & 29.638 & 0.09 & 1.55 & 1.55 & 2.61 \\
\hline & \multicolumn{4}{|c|}{$R(\mathrm{~mm}) k\left(\mathrm{~mm}^{-1}\right)$} & \multicolumn{4}{|c|}{$p_{\text {error }}(\mathrm{mm})$} \\
\hline Single & Ex07 & 250 & $4.29 \times 10^{-3}$ & 21.622 & 2.02 & 1.48 & 2.51 & 5.01 \\
\hline bend & Ex08 & 250 & $4.29 \times 10^{-3}$ & 21.622 & 2.23 & 1.34 & 2.60 & 5.44 \\
\hline \multicolumn{5}{|c|}{ Overall Results of $p_{\text {error }}(\mathrm{mm})$} & 0.68 & 1.45 & 1.61 & 5.44 \\
\hline
\end{tabular}
the $y$-axis position of the probe tip, respectively. As in (22),

TABLE 2.

EXPERIMENTAL RESULT OF PROBE STEERING (ERRORS TO ${ }^{\mathrm{B}} \mathrm{P}_{\mathrm{T}}$ ) 
TABLE 3.

TRACKING RESUlts PLACED IN THE CONTEXT OF PREVIOUS WORK

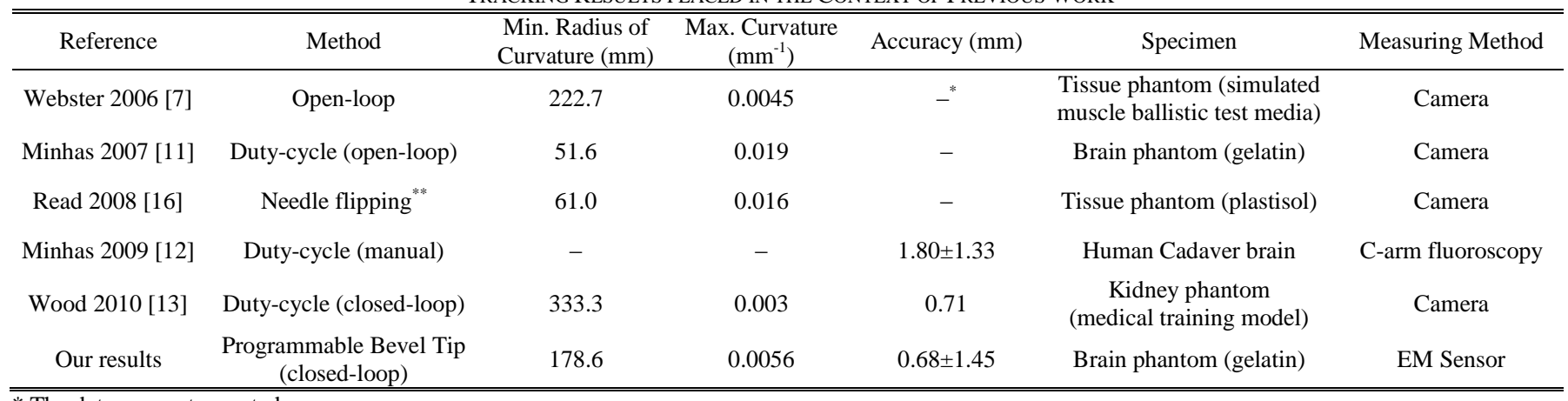

* The data was not reported.

** Needling flipping indicates the "on-off" controller, which switches between "bevel-right" and "bevel-left".

the gains utilized in these experiments favor the tracking of the curvature, not y-axis position tracking because $\left[k_{2}, k_{3}, k_{4}\right]=$ $[0.2,0.02,0.001]$. However, a series of simulation runs revealed limitations in the viable control gain range because of the constraint described in (37), which affects tracking convergence when the gains or the positional offset are large, as the constraint restricts the maximum changing rate of steering (since offset cannot change on the spot i.e. with the simultaneous forward motion of one steering segments and backward motion of the other). To highlight this limitation, simulation experiments without constraint (37) have shown that the probe can track the desired path robustly under a much wider range of initial conditions, but these results have not been reported here for brevity. Thus, the control strategy implemented will need to be refined to improve the changing rate of steering without breaking the constraint.

Limitations in the viable control gain range may also explain why the tracking errors for the single bend trajectories (i.e. Ex07 and Ex08) are larger than those for double bends, which seems counterintuitive. Since the feed-forward input does not vary significantly during the single-bend experiments, the tracking error is caused mainly by the small gains of the feedback controller. However, in the double-bend experiments, the feed-forward input changes significantly halfway into the trajectory (i.e. $x \approx 75 \mathrm{~mm}$ ), which causes the corresponding tracking error to reduce: the friction between segments pushes the whole body back as the steering offset gradually changes sign.

TABLE 3 summarizes how these experimental results compare to those previously reported in the literature. Even
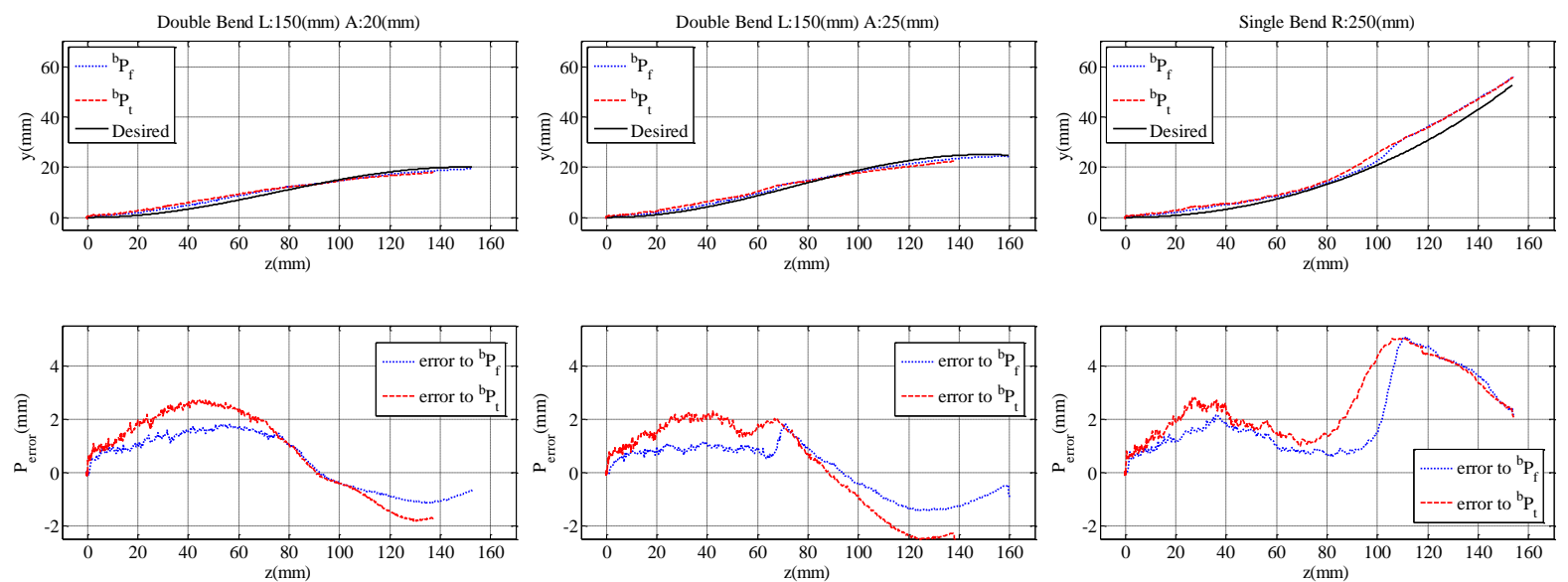

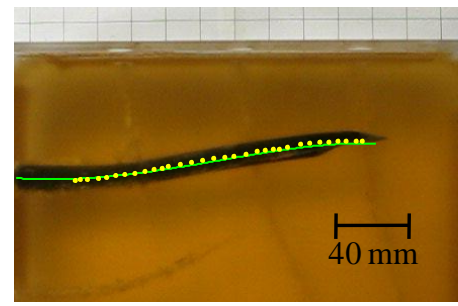

Ex04

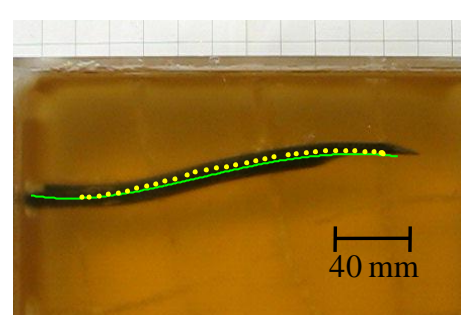

Ex06

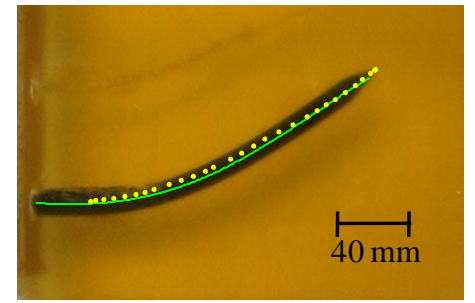

Ex07

Fig. 14. Experimental results of probe steering. The plots in the first row describe the trajectories achieved with the virtual probe tip ( ${ }^{\mathrm{B}} \mathrm{P}_{\mathrm{F}}$ ) estimated using (26) and (33), the tip of the segment with embedded EM sensor $\left({ }^{\mathrm{B}} \mathrm{P}_{\mathrm{T}}\right)$ and desired trajectory for three example paths (i.e. Ex04, Ex06 and Ex07; left to right). The plots in the second row show the positional error from the desired trajectory. The images in the bottom row depict actual trajectories of the flexible probe. The green sold lines illustrate the centre axes of the final shapes, while the yellow dotted lines indicate the trace of the virtual probe tip $\left({ }^{\mathrm{B}} \mathrm{P}_{\mathrm{F}}\right)$ at 5 second intervals from start to end. 
though the outer diameter of the probe and the material properties of the probe and the substrate will affect performance, the minimum radius of curvature and the accuracy achieved in our experiments are broadly in line with those obtained with other steering methods. Moreover, miniaturization of the current flexible probe prototype will potentially result in improved steering performance.

In addition, steering of our flexible probe only relies on the steering offset, which in turn only depends on the forward motion of each segment. Therefore, contrary to other research works [11-13, 16], the control strategy outlined here does not require rotation of the flexible probe around the long centre axis. When compared to the drilling effect of a rotating needle [39] or to the discontinuity introduced by flipping a flexible needle with a pre-bent tip [16], the proposed control approach is expected to cause less strain to the surrounding tissue and, in case of successful prototype miniaturization, less tissue damage.

\section{CONCLUSION \& FUTURE WORK}

This paper describes on-going research into the control of a bio-inspired flexible probe. Based on the characteristics of certain ovipositing wasps, a novel flexible probe with multiple segments, which are connected to each other by means of a special interlocking mechanism, was manufactured and the concept of a "programmable bevel" was proposed to steer the probe in a predefined direction: the offset between probe segments determines the steering direction of the tip thanks to a set of bevels and the amount of unsupported length associated with the leading segment. A kinematic model of the flexible probe was derived, based on its similarity to that of car-like robots. In this research, the curvature of the probe is assumed to be a function of the steering offset, which is shown empirically to be proportional to the steering offset through a simple set of experimental trials in gelatin. A compensation algorithm to account for differences in steering offset between base and tip coordinates was also proposed. Finally, a closedloop control strategy, which utilizes both feed-forward and feedback components, was implemented for two-dimensional trajectory following using an approximate linearization technique. In vitro experimental results demonstrate that both the kinematic model and the control method perform as expected.

Though these early experimental results are promising, research to date offers significant scope for future work. Specifically, further studies are planned on the miniaturization of the current prototype down to a clinically acceptable size (e.g. $\sim 2 \mathrm{~mm}$ OD for neurosurgery, $\sim 4 \mathrm{~mm}$ OD for breast biopsy [40] and drainage of liver abscesses [41]), a further reduction to the minimum radius of curvature and an investigation into the tip forces required for neurosurgery. It is worth noting that the size of the working channel resulting from a reduction in outer diameter may limit the range of applications (e.g. drug delivery) for which a miniaturized device would be suitable. However, it is expected that a 2-3 $\mathrm{mm}$ OD probe would be functionally similar to existing 25 gauge needles (i.e. with an inner diameter of $0.26 \mathrm{~mm}$ ) and that further optimization of the design (e.g. location of the working channel, number of independent segments) would allow us to further increase the size of the working channel for a given outer diameter size. In addition, tip positional tracking will also be challenging, as space for the position sensor will reduce with scale. However, the smallest EM sensor currently available is $0.5 \mathrm{~mm}$ in diameter and connecting wires are thinner than the sensor itself [37]. Thus, it is expected that EM-based position tracking of a miniaturized probe will be possible, although alternatives (e.g. intra-operative imaging based on magnetic resonance imaging and fluoroscopy) will also be considered. The inclusion of an additional sheath or canula, as suggested in [17], will also be considered as a means to further enhance the functionality of the probe. The manufacturing of a scaled-down device will thus be challenging, but early experience of extrusion with a medical grade silicon blend is promising. While an analysis of the performance of this further prototype falls outside the scope of this paper, it suggests that, although taxing, miniaturization of the probe is indeed possible.

Optimization of the closed-loop control approach described here is necessary to reduce tracking error and improve performance. This optimization may include further tuning of the control gains in light of existing constraints, which may result in changes to the algorithm which overcome the limitations on the changing rate of the steering angle (or offset). In the current implementation, we used the EM sensor as ground truth. However, it will be necessary to assess the performance of the flexible probe as a whole, including the effect of any sensor-induced inaccuracies. This study will be complemented by a detailed analysis of the rate- and substratedependent behavior of the probe. In addition, we currently assume that there is no friction between probe segments and that the probe is very flexible, while being stiff in compression and in tension. However, experiments to date on the prototype show that such assumptions may be overoptimistic, which means that further improvements to the kinematic model are needed. As a method to reduce the interaction forces between the probe and the tissue, a reciprocal insertion process, where each segment is inserted further into the tissue in turn, will also be considered as this approach has been shown to reduce the risk of buckling in insects [25]. Finally, both the kinematic model and control schemes described here will be extended to three-dimensions (3D). With the ability to actuate all four segments independently, combinations of simultaneous steering offsets in the two orthogonal planes and their effect on tip orientation will be explored. Development of a suitable 3D kinematic model and an extension of the current controller to $3 \mathrm{D}$ are thus planned.

\section{REFERENCES}

[1] N. Abolhassani, R. Patel, and M. Moallem, "Needle insertion into soft tissue: A survey," Medical Engineering \& Physics, vol. 29, pp. 413-431, 2007.

[2] S. P. DiMaio and S. E. Salcudean, "Needle Steering and Model-Based Trajectory Planning," in MICCAI, 2003. 
[3] D. Glozman and M. Shoham, "Flexible Needle Steering and Optimal Trajectory Planning for Percutaneous Therapies," Lecture Notes in Computer Science, vol. 3217, pp. 137-144, 2004.

[4] P. E. Dupont, Jesse Lock, B. Itkowitz, and E. Butler, "Design and Control of Concentric-Tube Robots," IEEE Transactions on Robotics, vol. 26, pp. 209-225, April 2010.

[5] D. C. Rucker, B. A. Jones, and R. J. Webster III, "A Model for Concentric Tube Continuum Robots Under Applied Wrenches," in IEEE International Conference on Robotics and Automation, Anchorage, Alaska, USA, 2010, pp. 1047-1052.

[6] L. A. Lyons, R. J. Webster III, and R. Alterovitz, "Planning Active Cannula Configurations Through Tubular Anatomy," in IEEE International Conference on Robotics and Automation, Anchorage, Alaska, USA, 2010.

[7] R. J. Webster III, J. S. Kim, N. J. Cowan, G. S. Chirikjian, and A. M. Okamura, "Nonholonomic Modeling of Needle Steering," The International Journal of Robotics Research, vol. 25, pp. 509-525, MayJune 2006.

[8] R. Alterovitz, M. Branicky, and K. Goldberg, "Motion Planning Under Uncertainty for Image-guided Medical Needle Steering," The International Journal of Robotics Research, vol. 27, pp. 1361-1374, November 12008.

[9] K. B. Reed, A. M. Okamura, and N. J. Cowan, "Modeling and Control of Needles with Torsional Friction," IEEE Transactions on Biomedical Engineering, vol. 56, pp. 2905-2916, 2009.

[10] V. Kallem and N. J. Cowan, "Image Guidance of Flexible Tip-Steerable Needles," IEEE Transactions on Robotics, vol. 25, pp. 191-196, February 2009.

[11] D. S. Minhas, J. A. Engh, M. M. Fenske, and C. N. Riviere, "Modeling of Needle Steering via Duty-Cycled Spinning," in the 29th Annual International Conference of the IEEE EMBS, Lyon, France, 2007, pp. pp. 2756-2759.

[12] D. Minhas, J. A. Engh, and C. N. Riviere, "Testing of Neurosurgical Needle Steering via Duty-Cycled Spinning in Brain Tissue in Vitro," in IEEE EMBS, Minneapolis, Minnesota, USA, 2009, pp. 258-261.

[13] N. A. Wood, K. Shahrour, M. C. Ost, and C. N. Riviere, "Needle Steering System using Duty-Cycled Rotation for Percutaneous Kidney Access," in International Conference of the IEEE EMBS, Buenos Aires, Argentina, 2010, pp. 5432-5435.

[14] K. Hauser, R. Alterovitz, N. Chentanez, A. Okamura, and K. Goldberg, "Feedback Control for Steering Needles Through 3D Deformable Tissue Using Helical Paths," in Robotics: Science and Systems Conference, Seattle, WA, 2009.

[15] D. Glozman and M. Shoham, "Image-Guided Robotic Flexible Needle Steering," IEEE TRANSACTIONS ON ROBOTICS, vol. 23, pp. 459-467, June 2007.

[16] K. B. Reed, V. Kallem, R. Alterovitz, K. Goldberg, A. M. Okamura, and N. J. Cowan, "Integrated Planning and Image-Guided Control for Planar Needle Steering," in IEEE/RAS-EMBS International Conference on Biomedical Robotics and Biomechatronics, Scottsdale, AZ, USA, 2008.

[17] A. Majewicz, T. R. Wedlick, K. B. Reed, and A. M. Okamura, "Evaluation of Robotic Needle Steering in ex vivo Tissue," in IEEE International Conference on Robotics and Automation, Anchorage, Alaska, USA, 2010, pp. 2068-2073.

[18] E. C. Burdette, D. C. Rucker, P. Prakash, C. J. Diederich, J. M. Croom, C. Clarke, P. Stolka, T. Juang, E. M. Boctor, and R. J. Webster III, "The ACUSITT Ultrasonic Ablator: The First Steerable Needle with an Integrated Interventional Tool " in Proceedings of SPIE Medical Imaging, San Diego, California, USA, 2010.

[19] T. Parittotokkaporn, L. Frasson, A. Schneider, S. E. Huq, B. L. Davies, P. Degenaar, J. Biesenack, and F. M. Rodriguez y Baena, "Soft Tissue Traversal with Zero Net Force: Feasibility Study of a Biologically Inspired Design Based on Reciprocal Motion," in IEEE International Conference on Robotics and Biomimetics, 2008, pp. 80-85.

[20] L. Frasson, T. Parittotokkaporn, A. Schneider, B. L. Davies, J. F. V. Vincent, S. E. Huq, P. Degenaar, and F. M. Rodriguez y Baena, "Biologically inspired microtexturing: Investigation into the surface topography of next-generation neurosurgical probes," in 30th Annual International Conference of the IEEE Engineering in Medicine and Biology Society, 2008, pp. 5611-5614.

[21] L. Frasson, S. Y. Ko, A. Turner, T. Parittotokkaporn, J. F. Vincent, and F. Rodriguez y Baena, "STING: a soft-tissue intervention and neurosurgical guide to access deep brain lesions through curved trajectories," Proceedings of the Institution of Mechanical Engineers,
Part H: Journal of Engineering in Medicine, vol. 224, pp. 775-788, 2010.

[22] L. Frasson, S. Reina, B. L. Davies, and F. M. Rodriguez y Baena, "Design Optimization of a Biologically Inspired Multi-Part Probe for Soft Tissue Surgery," in 11th International Congress of the IUPESM, Medical Physics and Biomedical Engineering, Munich, German, 2009, pp. 307-310.

[23] S. Y. Ko, B. L. Davies, and F. Rodriguez y Baena, "Two-Dimensional Needle Steering with a "Programmable Bevel" Inspired by Nature: Modeling Preliminaries," in IEEE/RSJ International Conference on Intelligent Robots and Systems, Taipei, Taiwan, 2010, pp. 2319-2324.

[24] L. Vilhelmsen, N. Isidoro, R. Romani, H. H. Basibuyuk, and D. L. Quicke, "Host location and oviposition in a basal group of parasitic wasps: the subgenual organ, ovipositor apparatus and associated structures in the Orussidae (Hymenoptera, Insecta)," Zoomorphology, vol. 121, pp. 63-84, 2001.

[25] J. F. V. Vincent and M. J. Kin, "The Mechanism of Drilling by Wood Wasp Ovipositors," Biomimetics, vol. 3, pp. 187-201, 1995.

[26] D. L. J. Quicke and M. G. Fitton, "Ovipositor steering mechanisms in parasitic wasps of the families Gasteruptiidae and Aulacidae (Hymenoptera)," Proceedings of The Royal Society B-Biological Sciences vol. 261, pp. 99-103, 1995.

[27] D. L. Quicke, A. L. Ralec, and L. Vilhelmsen, "Ovipositor structure and function in the parasitic Hymenoptera with an exploration of new hypotheses," Atti della Accademia Nazionale Italiana di Entomologia Rendiconti, vol. 47, pp. 197-239, 1999.

[28] M. Oldfield, D. Dini, and F. Rodriguez y Baena, "Detailed finite element simulations of probe insertion into solid elastic material using a cohesive zone approach," in International Conference of the IEEE Engineering in Medicine and Biology Society, Buenos Aires, Argentina, 2010, pp. 31983201.

[29] A. De Luca, G. Oriolo, and C. Samson, "Feedback Control of a Nonholonomic Car-like Robot " in Robot Motion Planning and Control, J. P. Laumond, Ed.: Springer, 1998, pp. 172-253.

[30] E. J. Hearn, Mechanics of materials. 1, An introduction of the mechanics of elastic and plastic deformation of solids and structural materials, 3rd ed. ed. Oxford: Butterworth-Heinemann, 1997.

[31] R. M. Murray and S. S. Sastry, "Nonholonomic Motion Planning: Steering Using Sinusoids," IEEE TRANSACTIONS ON AUTOMATIC CONTROL, vol. 38, pp. 700-716, May 1993.

[32] C. Samson, "Control of Chained Systems Application to Path Following and Time-Varying Point-Stabilization of Mobile Robots," IEEE Transactions on Automatic Control, vol. 40, pp. 64-77, January 1995.

[33] B. d'Andréa-Novel, G. Campion, and G. Bastin, "Control of Nonholonomic Wheeled Mobile Robots by State Feedback Linearization " The International Journal of Robotics Research, vol. 14, pp. 543-559, 1995.

[34] J.-M. Yang and J.-H. Kim, "Sliding mode control for trajectory tracking of nonholonomic wheeled mobile robots," IEEE Transactions on Robotics and Automation, vol. 15, pp. 578-587, June 1999.

[35] R. S. Sharp, "Optimal linear time-invariant preview steering control for motorcycles," Vehicle System Dynamics, vol. 44, Supplement, pp. 329340,2006

[36] Y. Tan, Z. Jiang, and Z. Zhou, "A Nonholonomic Motion Planning and Control Based on Chained Form Transformation," in IEEE/RSJ International Conference on Intelligent Robots and Systems, Beijing, China, 2006, pp. 3149 - 3153.

[37] Northern Digital Inc., (2010, Feb 19), Available: http://www.ndigital.com/

[38] D. K. Binder, G. M. Rau, and P. A. Starr, "Risk Factors For Hemorrhage During Microelectrode-guided Deep Brain Stimulator Implantation For Movement Disorders," Neurosurgery, vol. 56, pp. 722-732, April 2005.

[39] M. A. Meltsner, N. J. Ferrier, and B. R. Thomadsen, "Observations on rotating needle insertions using a brachytherapy robot," Physics in Medicine and Biology, vol. 52, pp. 6027-6037, 2007.

[40] J.-S. He, X.-M. Wang, S.-C. Chen, W.-C. Chen, S.-F. Huang, Y.-H. Liu, J.-T. Zhang, Z.-Y. Li, W.-Z. Liu, and H. Guan, "Evaluation on pathologic status of the residual lesions after ultrasound-guided, 8-gauge vacuum-assisted mammotome ${ }^{\circledR}$ system biopsy for nonpalpable malignant breast lesions and its influencing factors," Scientific Research and Essays, vol. 5, pp. 3467-3473, 2010.

[41] C. L. Rajak, S. Gupta, S. Jain, Y. Chawla, M. Gulati, and S. Suri, "Percutaneous Treatment of Liver Abscesses: Needle Aspiration Versus Catheter Drainage," American journal of roentgenology, vol. 170, pp. 1035-1039, April 1998. 


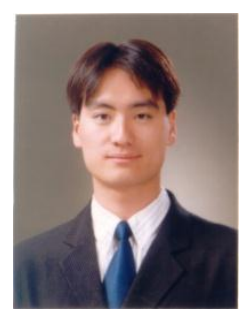

Seong Young Ko (M'10) received the B.S. degree, the M.S. degree, and the Ph.D. degree in Mechanical Engineering from Korea Advanced Institute of Science and Technology (KAIST), Korea, in 2000, 2002, and 2008, respectively. He was a visiting researcher in the Department of Electrical Engineering, University of Washington, USA for 6 months from 2005 to 2006. In 2008, he was a post-doctoral researcher in the Department of Electrical Engineering, KAIST, Korea. From 2009, he is now a research associate in the Mechatronics in Medicine Laboratory, the Department of Mechanical Engineering, Imperial College London, UK. His research interests include a medical robotics, human-robot interaction and intelligent control.

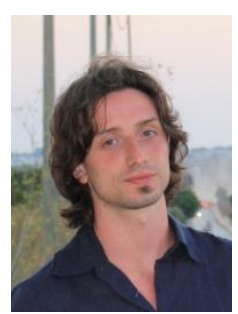

Luca Frasson received the Bachelor and Master degrees in Biomedical Engineering from Politecnico di Milano, Italy, in 2004 and 2006, respectively. He then gained a PhD in Medical Robotics in the Department of Mechanical Engineering at Imperial College of London, in 2010. He is now a patent consultant. His research interests include medical robotics for neurosurgery, biomimetics, and wearable sensors.

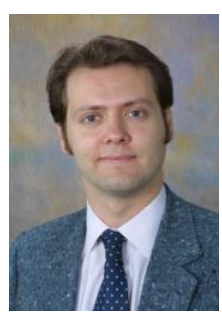

Ferdinando Rodriguez y Baena (M'09) graduated with a First Class Honours degree in Mechatronics and Manufacturing Systems Engineering from King's College London in 2000. He then joined the Department of Mechanical Engineering at Imperial College, where he gained a PhD in Medical Robotics. He is now a Senior Lecturer in the same Department, where he leads the Mechatronics in Medicine Laboratory. His current research interests lie in the application of mechatronic systems to medicine, in the specific areas of clinical training, diagnostics and surgical intervention. 